\title{
ANÁLISIS DE FALLA POR FATIGA EN EL EJE CONDUCIDO DE LA BOMBA HILLMAN 250B MTB PARA EL TRANSPORTE DE CRUDO
}

\section{ANALYSIS OF FATIGUE FAILURE OF THE SHAFT DRIVEN PUMP HILLMAN 250B MTB FOR TRANSPORT OIL}

\author{
MSc. Ricardo Andrés García-León" ***, MSc. Edwin Edgardo Espinel Blanco* \\ MSc. Eduar Pérez Rojas* \\ *Universidad Francisco de Paula Santander Ocaña, \\ Facultad de Ingenierías, Grupo de Investigación INGAP. \\ Vía Acolsure, Sede el Algodonal - Ocaña, Norte de Santander, Colombia. \\ Tel.: +57 5690088 Ext. 212 - 191. \\ E-mail: (ragarcial, eeespinelb, eeperezr) @ufpso.edu.co. \\ ** Instituto Politécnico Nacional, \\ Grupo Ingeniería de Superficies, SEPI-ESIME, U.P. Adolfo López Mateos, Zacatenco, \\ Ciudad de México 07738, México
}

Resumen: El objetivo del presente estudio, está centrado en localizar las causas que originaron la falla por fatiga en el eje conductor de la bomba Hillman 250B MTB doble tornillo para el despacho de crudo. El eje tuvo fractura en el cuñero cerca de la zona de cambio de sección debido a malos procedimientos ingenieriles sin tener en cuenta los cálculos de diseño. Por esta razón, se realizó un análisis de causa raíz con el cual se demostró que el cuñero estaba mal diseñado, y de esta manera poder establecer las variables que influyeron en la falla. Luego de obtener esta información, se procedió a realizar el análisis del diseño óptimo del cuñero, igualmente el rediseño del concentrador de esfuerzo para evitar futuras fallas, mediante un análisis de fatiga, fuerzas pares y diagramas de esfuerzos cortante para materiales dúctiles del cual estaba fabricado, con lo que se determinó la vida del eje. Además, se efectuó el modelamiento por medio de elementos finitos para determinar el estado de los esfuerzos. Asimismo con los resultados obtenidos poder presentar los planes de acción para evitar futuras fallas tempranas en los sistemas de bombeo principales para el despacho de crudo en las empresas del sector petrolero.

Palabras clave: Bomba; Crudo; Falla; Fatiga; Eje; Fuerzas.

\begin{abstract}
The aim of this study is focused on locating the causes of fatigue failure on the drive shaft of the pump twin screw MTB 250B Hillman for the release of oil. The shaft had broken keyway area near the section change due to poor engineering procedures regardless design calculations. For this reason, a root cause analysis with which it was shown that the keyway was poorly designed, and thus to establish the variables that influenced the failure was performed. After obtaining this information, we proceeded to the analysis of the optimal design of the keyway, also redesigning the hub effort to avoid future failures, through an analysis of fatigue, even forces and diagrams of shear stresses for ductile materials which was made, which shaft life was determined. Furthermore, modeling was performed using finite elements to determine the state of stress. Likewise with the results obtained to present action plans to prevent future early failures main pumping systems for the release of oil in the oil sector companies.
\end{abstract}

Keywords: Pump; Raw; Failure; Fatigue; Axis; Forces. 


\section{INTRODUCCIÓN}

Las bombas doble tornillo son implementadas para el bombeo de cualquier tipo de lubricante o fluido, utilizadas en gran parte en el sector petrolero para el bombeo de crudo debido a sus óptimas características de funcionamiento y mantenimiento. Estas bombas están compuestas principalmente por un eje tornillo conductor que acciona el eje conducido mediante un engranaje de sincronización que asegura la rotación simultánea de los tornillos lo que produce el transporte del fluido (Piovan, 2014) (Marco Esteban, 2010) (De Vedia \& Svodoba, 2002) (Casanova, 2010).

La desviación del eje y la distribución de la carga asimétrica, hace que los ejes fallen con frecuencia. Por lo cual se realiza el análisis del eje aplicando la fórmula para la amplitud de tensión en donde el esfuerzo equivalente varía linealmente con la carga correspondiente, lo que generalmente produce las fallas en este tipo de componentes mecánicos (X. J. Li, Hu, Liu, \& Chen, 2004). Waldon en un artículo, revisa los resultados preliminares de un proyecto destinado a desarrollar técnicas para la evaluación horizontal de eje con la finalidad de determinar la vida por fatiga del sistema (Waldon, 1983).

Teniendo en cuenta lo anterior, en muchas investigaciones se han evaluado los mecanismos de daño producido por una fibra de carbono unidireccionalmente en compuestos reforzado de una matriz metálica de bajo la carga cíclica sobre el eje. En la variación de la fatiga se puede encontrar que la vida del eje depende de la microestructura y la facilidad con la que tuvo lugar la coalescencia de microgrietas en la matriz. Cuando grietas en la matriz se generan rápidamente, la fatiga de la vida es relativamente corta.

Y. Shen y S. Wang, en su estudio realizaron el análisis multiaxial de la vida por fatiga de un rodillo en contacto de rodadura con ruedas, teniendo en cuenta la desviación de línea del eje. Los análisis de estrés se realizaron dentro del análisis de elementos finitos con el software ANSYS. La tensión de fricción tangencial se calcula en términos de la condición de la zona de contacto de rodadura (Shen, Wang, Li, \& Dhillon, 2010), así mismo se analizó el eje principal para determinar la fatiga y el coeficiente de uso de la vida y poder evaluar los requisitos de trabajo. (C. X. Li, Hu, Dai, \& Bian, 2009) (Vachon, 1988)
Otros análisis de laboratorio que se pueden realizar en ejes o componentes de máquinas, es la Microscopía electrónica de barrido combinada con FIB de corte y formación de imágenes EBSD en las secciones longitudinales que contienen grietas con el objetivo de encontrar los mecanismos responsables de la fatiga iniciación de grietas y su crecimiento. (Petráš, Škorík, \& Polák, 2016)

\section{MATERIALES Y MÉTODOS}

Con el fin de proceder con el desarrollo del análisis de falla en el eje conducido de la bomba de tornillo HILMAN DESPACHO DE CRUDO 250B MTB (ver Figura 1), se desarrollaron una serie de cálculos matemáticos y análisis por elementos finitos mediante el software ANSYS, teniendo en cuenta las características del eje, con la finalidad de determinar las posibles causas de falla. Para realizar este procedimiento se deben tener en cuenta las observaciones del reporte de la falla generado por el profesional de confiabilidad que hace parte de la empresa, el cual es el encargado de garantizar el correcto funcionamiento de los equipos.

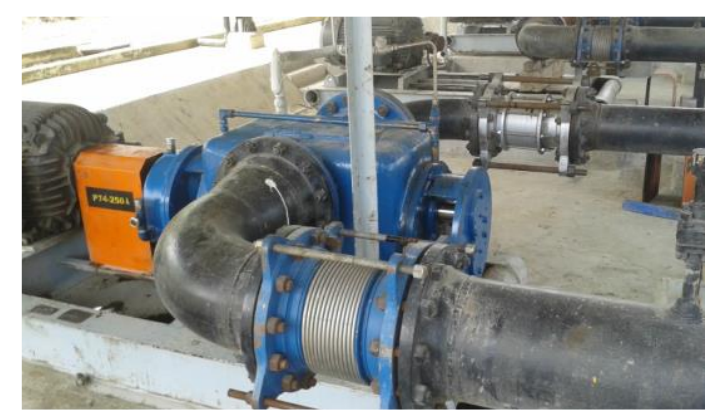

Fig. 1. Bomba de tornillo hillman.

Luego de realizar una inspección visual se detallan las condiciones de maquinado de la superficie en el montaje de los piñones del eje conductor, los cuales son diferentes a las del eje del piñón conducido (ver figura 2).

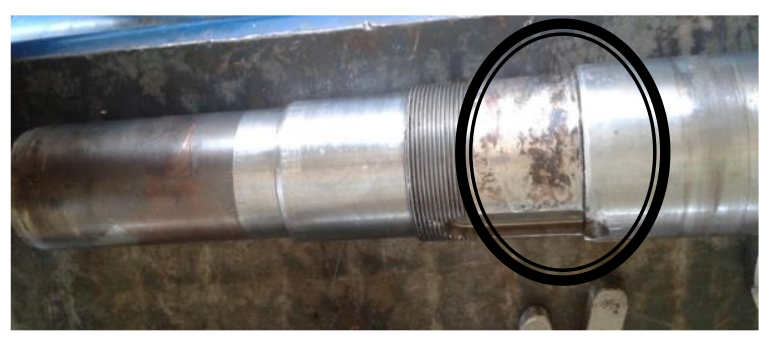

Fig. 2. Condición de la superficie del montaje del piñón conductor.

Seguidamente, se puede observar que la fatiga en el material inicia a un costado del cuñero que actúa 
como concentrador de esfuerzo cuando el piñón le transmite la carga al eje (ver Figura 3).

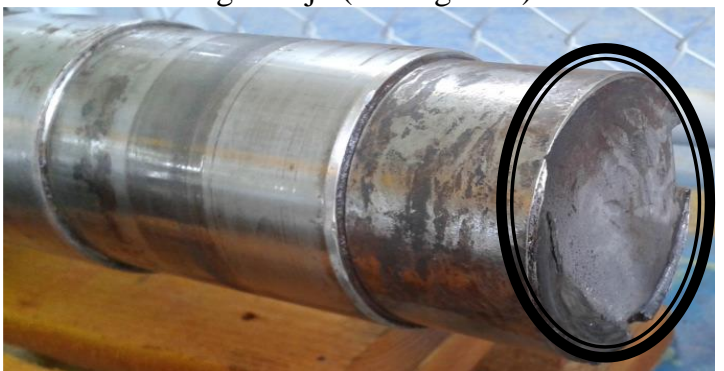

Fig. 3. Inicio de la fatiga.

Además, se evidencia que el eje fue reconstruido y se presenta un cambio de sección diferente al eje original ya que este poseía un radio de curvatura interno y se observa que este radio cambio de cóncavo a convexo (ver figura 4).

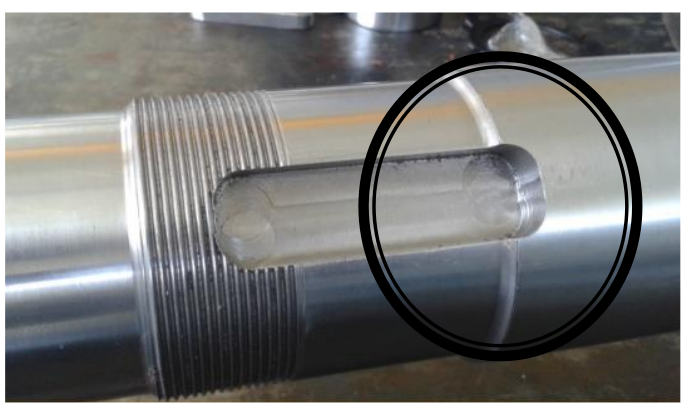

Fig. 4. Cambio de sección.

También se determinó que el diseño del cuñero no satisface la geometría de la cuña, lo que genera un desfase entre estos elementos generando vibraciones en eje (ver figura 4).

Una vez con el reporte del profesional de confiabilidad se inicia el análisis de falla mediante el árbol de fallas, y de esta manera poder determinar la causa raíz de la falla en el eje para proceder a revisar los parámetros de diseño del mismo (ver figura 5).

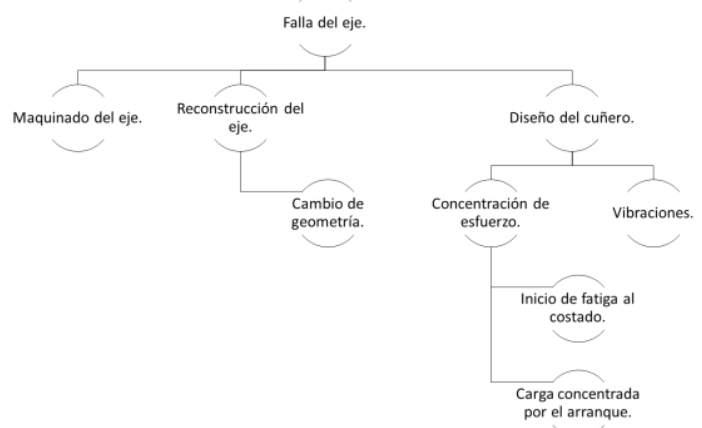

Fig. 5. Análisis causa raíz.

\section{RESULTADOS Y DISCUSIÓN}

Universidad de Pamplona

I. I. D. T. A.
En esta sección se presentan los resultados obtenidos mediante los cálculos realizados puntos críticos del eje, siendo estos indispensables para la construcción y buen funcionamiento del elemento mecánico, con el fin de tener un análisis verás mediante cálculos matemáticos para lo cual, es necesario conocer las propiedades mecánicas del material y su geometría.

Los cálculos que se tienen en cuenta para el desarrollo de la investigación, se basan en el análisis de diseño para comprobar que se cumplan con los requerimientos que demandan las cargas a las que se ve sometido el eje. A continuación se detalla la geometría y los puntos de falla del eje (ver figura 6).

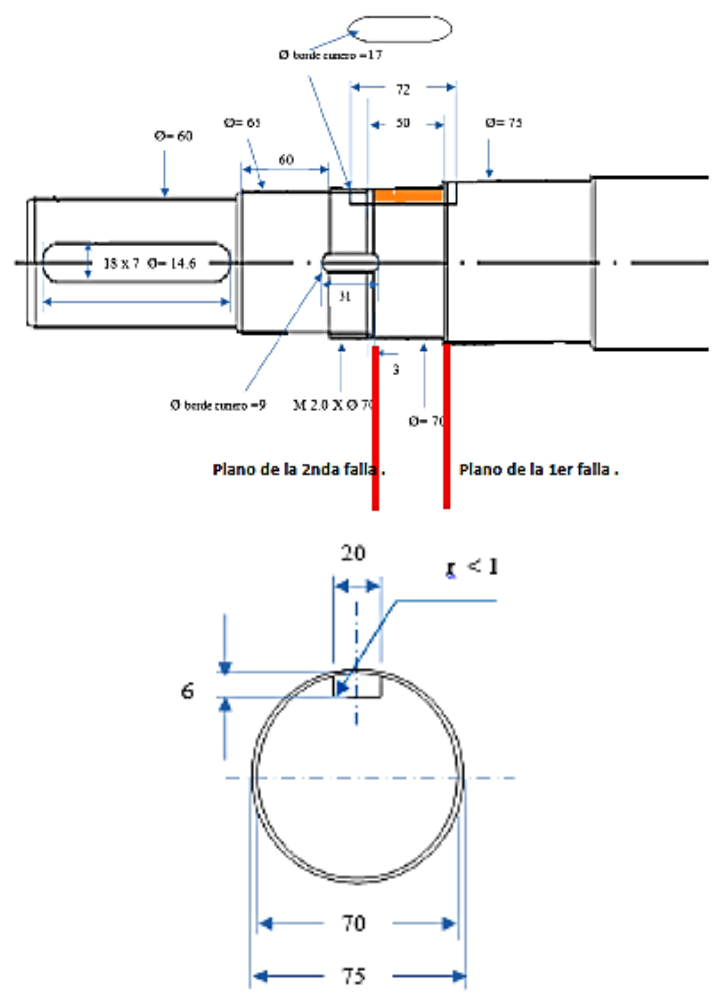

Fig. 6. Geometría del eje.

Luego de determinar que las fallas se dan por fatiga y los parámetros de diseño del eje que han sido modificados en su geometría en lo que respecta a la reconstrucción que fue sometido el eje, se establece que se debe realizar el análisis de resistencia a la fatiga del material mediante los siguientes cálculos:

\subsection{Análisis de los cuñero.}

Para iniciar el análisis se estipula como primer paso determinar las geometrías por catálogo de los cuñeros actuales, en donde se presenta la concentración de esfuerzo como se observa en la 
(figura 7), debido a que los cuñeros actuales se seleccionaron sin tener en cuenta parámetros de diseño, se determina que se deben modificar las características de la cuna y el cuñero basados en catálogos de diseño, con el fin de garantizar su correcta puesta en funcionamiento.
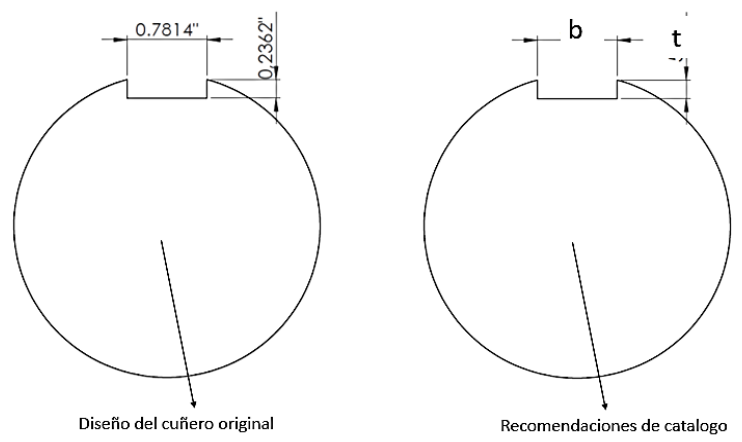

Fig. 7. Diseño de cuñeros.

Posteriormente a la observación de los cuñeros, se procede a determinar el desfase que se presenta en el cuñero mediante las siguientes Ecuaciones (1) y (2).

$\mathrm{b}=\frac{d}{4}$
$\mathrm{t}=\frac{d}{8}$

Dónde:

$b=$ Ancho del cuñero (In)

$t=$ Profundidad del cuñero (In)

$\mathrm{d}=$ diametro del eje (In)

Además, se determinó que la geometría del cuñero no es la recomendada puesto que se presentan desfases entre las longitudes recomendadas y las del eje, que según parámetros de diseño deben ser como mínimo de $12,5 \%$ para el ancho y $45 \%$ para la profundidad del cuñero, con lo cual se determina que al momento del arranque del motor se presenta un impacto por la carga de la cuña al eje.

\subsection{Geometría del elemento diferencial}

Teniendo ya identificado que el elemento diferencial es el punto donde se dio el inicio de la fatiga, se procede a analizar la geometría.

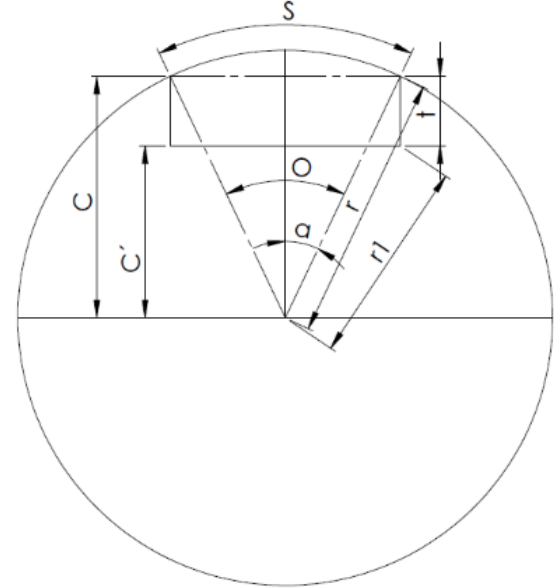

Fig. 8. Geometría del elemento diferencial.

\subsection{Esfuerzos por torsión.}

Luego de determinar las características geométricas del eje y del elemento diferencial, se establecen los esfuerzos cortantes que llevaron a la falla el eje (Figura 8), para ello se calculan las torques máximas y mínimas con la Ecuación (3).

$\mathrm{T}=\frac{5252 H}{n}$

Dónde:

$\mathrm{T}=$ Torque del motor $(\mathrm{Lb}-\mathrm{Ft})$

$\mathrm{H}=$ Potencia del Motor ( $\mathrm{Hp})$

$\mathrm{H}_{\mathrm{Max}}=300(\mathrm{Hp})$

$\mathrm{H}_{\min }=118(\mathrm{Hp})$

$\mathrm{n}=$ Numero de revoluciones del motor (Rpm)

Al calcular los torques máximos y mínimos del eje, se obtienen los siguientes:

$$
T_{\text {Max }}=1324(\mathrm{Lb}-\mathrm{Ft})
$$$$
T_{\min }=521(\mathrm{Lb}-\mathrm{Ft})
$$

Los cuales son utilizados para evaluar los esfuerzos cortantes en los elementos diferenciales A y C como se muestra (Figura 9) mediante la Ecuación 4. 


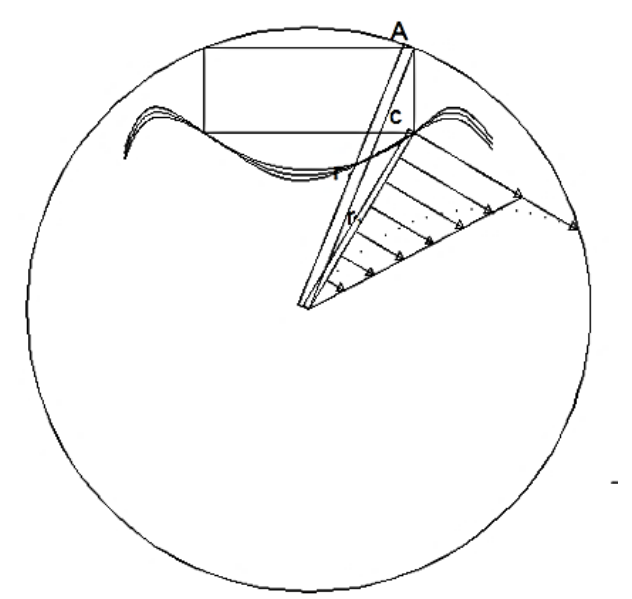

Fig. 9. Diagrama de esfuerzos por torsión.

$\tau=\frac{16 T}{\pi d^{a}}$

Dónde:

$\tau=$ Esfuerzo por torsion (Ksi)

$\mathrm{T}=$ Torque del motor $(\mathrm{Lb}-\mathrm{Ft})$

$\mathrm{d}_{\mathrm{A}}=2,750$ (In)

$\mathrm{d}_{\mathrm{C}}=1,596(\mathrm{In})$

De lo que se obtiene como resultado que los esfuerzos en los elementos diferenciales A y $\mathrm{C}$ son.

$\tau_{A}=3,8 \mathrm{Ksi}$

$\tau_{C}=20 \mathrm{Ksi}$

Para encontrar los esfuerzos máximos se multiplican los esfuerzos resultantes por el factor de carga máxima a bajos ciclos según las recomendaciones de diseño $K_{\operatorname{Max}}=1,45$, $\tau_{\text {AMax }}=5,51 \mathrm{Ksi} \tau_{\text {CMax }}=29 \mathrm{Ksi}$.

\subsection{Análisis de fuerzas y pares.}

En esta parte se determinan los esfuerzos normales del ciclo de trabajo y de arranque-parada del motor (Figura 10).

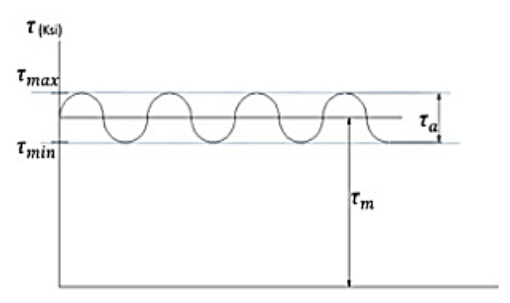

Ciclos oscilantes A torsión

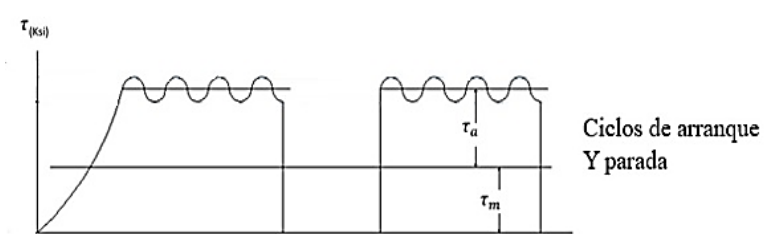

Fig. 10. Diagrama de ciclos.
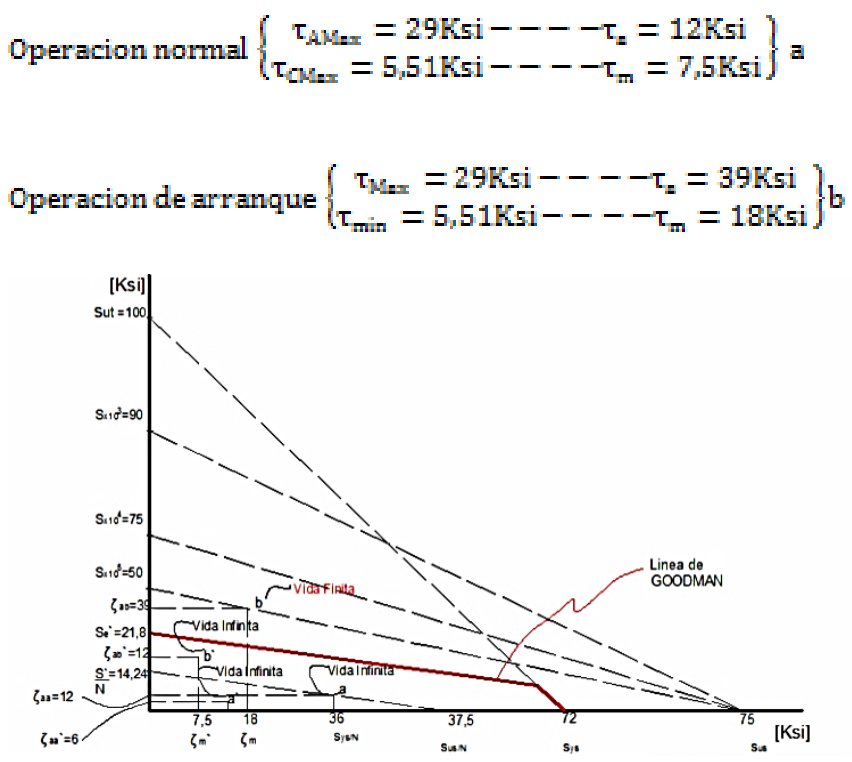

Fig. 11. Diagrama de esfuerzos para material dúctil.

Donde:

Sut $=$ Resistencia Máxima.

Sus $=$ Resistencia Máxima Cizalladura.

Syt $=$ Resistencia Máxima a la Tracción .

Sys $=$ Resistencia de Fluencia en Torsión.

$\mathrm{N}=$ Factor de seguridad.

Para calcular cada uno de los valores de entrada para el análisis de diseño, estos se encontraron con la información suministrada por el profesional de confiabilidad y con la base de datos que se muestra en las Tablas 1, 2, 3 y 4.

Tabla 1: Datos de entrada Concentrador de

\begin{tabular}{cccc}
\hline \multicolumn{4}{c}{ esfuerzo. } \\
\hline $\begin{array}{c}\text { Acabado } \\
\text { superficial }\end{array}$ & $\begin{array}{c}\text { Sut } \\
\text { (Kpsi) }\end{array}$ & $\begin{array}{c}\text { Sut } \\
\text { (MPa) }\end{array}$ & $\begin{array}{c}\text { Exponente } \\
\text { b }\end{array}$ \\
\hline Esmerilado & 1.34 & 1.58 & -0.085 \\
Maquinado & 2.7 & 4.51 & -0.265 \\
Laminado & 14.4 & 57.7 & -0.718 \\
Forjado & 39.9 & 272 & -0.995 \\
\hline
\end{tabular}


Tabla 2: Datos de entrada.

\begin{tabular}{ll}
\hline Variable & Valor \\
\hline Diámetro & $70 \mathrm{~mm}$ \\
Potencia Máxima & $223.71 \mathrm{Hp}$ \\
Potencia Mínima & $88 \mathrm{Hp}$ \\
Revoluciones & $1190 \mathrm{rpm}$ \\
Torque de diseño & 1796.45 \\
Temperatura de operación & $40^{\circ} \mathrm{C}$ \\
\hline
\end{tabular}

Tabla 3: Propiedades del material AISI 420.

\begin{tabular}{lrr}
\hline \multicolumn{1}{c}{ Variable } & \multicolumn{1}{c}{ Pa } & \multicolumn{1}{c}{ PSI } \\
\hline E & 200000000000 & 29007547546 \\
G & 77000000000 & 11168000 \\
Sut & 690000000 & 250000 \\
Sus & 518000000 & 75000 \\
Syt & 860000000 & 230000 \\
Sys & 496000000 & 72000 \\
\hline
\end{tabular}

Tabla 4: Datos para cálculos.

\begin{tabular}{lc}
\hline & Diámetro del Eje \\
\hline Diámetro 1 & $75 \mathrm{~mm}$ \\
Diámetro 2 & $70 \mathrm{~mm}$ \\
& Medidas de la Cuña \\
\hline b & $0.7874 \mathrm{~mm}$ \\
t & $0.2362 \mathrm{~mm}$ \\
Radio de muesca (r) & $2.5 \mathrm{~mm}$ \\
\hline
\end{tabular}

Teniendo en cuenta lo anterior, se planeó la siguiente geometría para el elemento diferencial:

$$
\begin{aligned}
& S=r e=0.57 \text { sabiendo que: } e=b / r \\
& \propto c=180 * \frac{e}{2 \pi}=16.41 \\
& c=r * \cos \propto c=1.319 \\
& c^{\prime}=c-t=0.694
\end{aligned}
$$

$\mathrm{r}_{1}=\sqrt{\mathrm{c}^{\mathrm{c} 2}+\left(\frac{\mathrm{b}}{2}\right)^{2}}=0.7979$

Basados en los resultados de la geometría del elemento, se recomiendan las siguientes especificaciones para el cuñero:

$$
\begin{aligned}
& \frac{\mathrm{v}}{\mathrm{d}}=1.0714 \\
& \frac{\mathrm{d}}{\mathrm{D}}=0.933
\end{aligned}
$$

Con las siguientes medidas, teniendo en cuenta las ecuaciones 1 y 2 : $\mathrm{b}=17.5$

$\mathrm{t}=8.75$

Con estas especificaciones, se aumenta la confiabilidad en un $99 \%$, obteniendo el siguiente valor de resistencia a la fatiga:

$$
\mathrm{Se}=\mathrm{Ks} * \mathrm{Kd} * \mathrm{Kt} * \mathrm{Kc} * \mathrm{Km} * \mathrm{Kts}=2.17 \times 10^{7}
$$

Sabiendo que:

$$
\begin{aligned}
& \mathrm{Ks}=\text { Factor de acabado superficial }=0.528 \\
& \mathrm{Kd}=\text { Factor de tamaño }=0.8456 \\
& \mathrm{Kt}=\text { Factor de temperatura }=1.001 \\
& \mathrm{Kc}=\text { Factor de Confiabilidad }=0.814 \\
& \mathrm{Km}=\text { Factores misceláneos }=0.84 \\
& \mathrm{Kts}=\text { Factor de esfuerzo por fatiga }=1.5
\end{aligned}
$$

EL cual presenta una confiabilidad del $50 \%$ de $\mathrm{Kc}=1$ y para el $100 \%$ de $\mathrm{Kc}=0.62$. Como se puede observar en la figura 10 y el análisis causa raíz, la falla se da en el eje por la concentración de esfuerzo del elemento diferencial definido como " $C$ " ya que este no se diseñó de una manera correcta.

Posteriormente al análisis de diseño para determinar la causa de la falla en el eje, se realizó un análisis de cargas torsional mediante modelado de elementos finitos con ANSYS, con los cuales se validaron los resultados obtenidos por el modelo matemático (García-León, R. A., Flórez-Solano, E. N., \& Acosta, M. A. 2015) (García-León, R. A., Flórez-Solano, E. N., \& Escobar-Macea, M. 2016) (García-León, R. A., Flórez-Solano, E. N., \& Sánchez-Ortiz, E. 2017). El cual arrojo los siguientes resultados en las Figuras 12 y 13 .

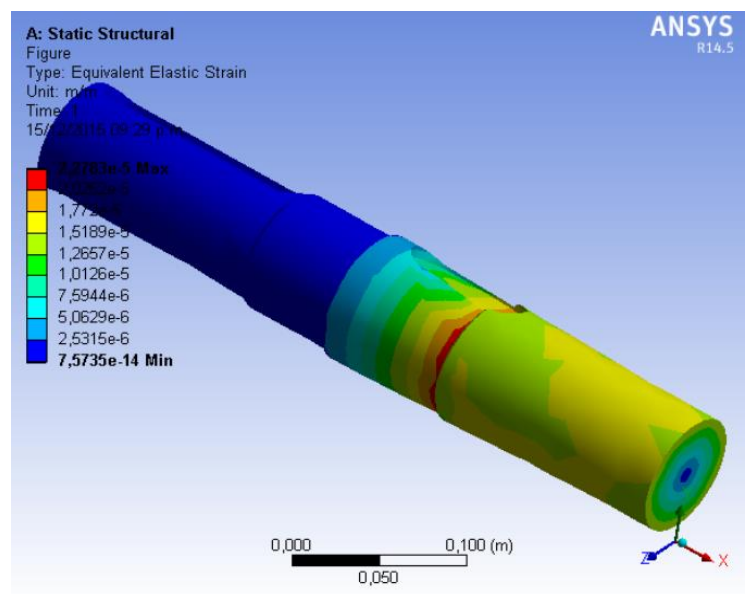

Fig. 12. Deformaciones elásticas. 
En la siguiente Figura 13, se muestran las deformaciones elásticas y unitarias que sufre el eje al ser sometido a las cargas de torsión que llevan a la falla por la concentración de esfuerzo por el mal diseño del cuñero.

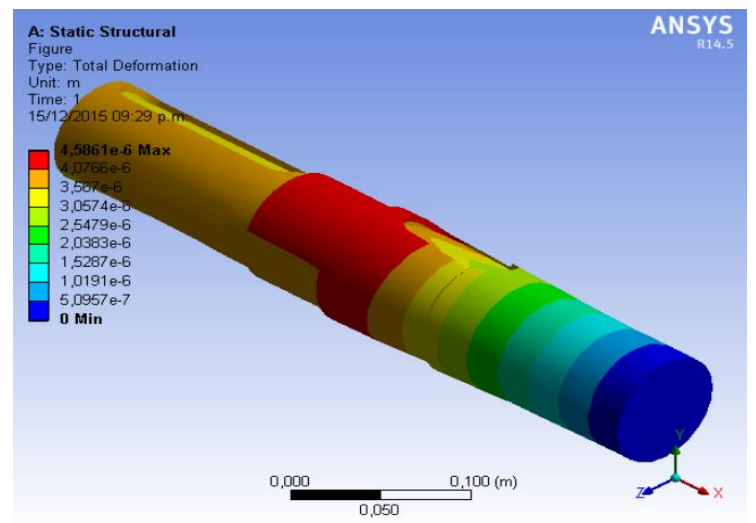

Fig. 13. Deformaciones totales.

Teniendo en cuenta estos resultados, se puede evidenciar que el eje presenta una concentración de esfuerzos en la zona donde se encuentra ubicado el cuñero, razón por la cual este debe ser diseñado de forma óptima para garantizar la correcta funcionalidad del sistema en términos de factor de seguridad y confiablidad.

\section{CONCLUSIONES}

Dentro del análisis causa raíz se pudo evidenciar que las modificaciones realizadas al eje sin un previo estudio de diseño causaron la falla en el concentrador de esfuerzo, también se denota que la modificación en el cambio de sección que paso de ser cóncavo a convexo genera una mayor concentración de esfuerzo.

Gracias al análisis de rediseño se evidencio que se debe tener en cuenta un criterio profesional para efectuar modificaciones en los ejes ya que la experiencia empírica no es confiable.

Se recomienda modificar el cuñero con criterios de diseño del mismo para evitar que la carga se concentre en el elemento diferencial y así disminuir la probabilidad de falla, como se muestra en la Figura 13, debido a que dicha modificación genera mayor confiabilidad en el elemento diferencial.

\section{REFERENCIAS}

Casanova, F. (2010). Failure investigation of the gear shaft connected to a double - screw extruder. DYNA (Colombia), 88-97.

De Vedia, L. A., \& Svodoba, H. (2002). Fatiga. In Ensayos Industriales (Buenos Air).

García-León, R. A., Flórez-Solano, E. N., \& Acosta, M. A. (2015). Análisis estructural de una maquina prensadora para la producción de ladrillo macizo para las pequeñas industrias artesanales de materiales cerámicos en Ocaña Norte de Santander y la región. Revista Colombiana de Tecnologías de Avanzada, 1(25), 104109.

García-León, R. A., Flórez-Solano, E. N., \& Escobar-Macea, M. (2016). Modelo de equipo de prensado tipo palanca, para mejorar la producción de queso en la provincia de Ocaña. Revista Colombiana de Tecnologías de Avanzada, 2(28), 140-149.

García-León, R. A., Flórez-Solano, E. N., \& Sánchez-Ortiz, E. (2017). Diseño de un sistema alimentador para un horno rotatorio en la producción de fosfato en el Norte de Santander. Revista Colombiana de Tecnologías de Avanzada, 1(29), 10-80.

Li, C. X., Hu, W. T., Dai, Z. B., \& Bian, X. (2009). Wind-induced fatigue analysis of main-shaft in a vertical-axis wind turbine. Zhendong $\mathrm{Yu}$ Chongji/Journal of Vibration and Shock, 28(7). Retrieved from http://www.scopus.com/inward/record.url?ei $\mathrm{d}=2-\mathrm{s} 2.0$ 68249114684\&partnerID=tZOtx3y 1

Li, X. J., Hu, R., Liu, D. S., \& Chen, G. Q. (2004). Relation between axis deviation and fatigue strength of supporting system of large-scale statically indeterminate rotary kiln. Gongcheng Lixue/Engineering Mechanics, 21(4), 51-56. Retrieved from http://www.scopus.com/inward/record.url?ei $\mathrm{d}=2-\mathrm{s} 2.0-6944232249 \&$ partnerID=tZOtx3y1

Marco Esteban, E. (2010). Metodología para el análisis a fatiga mediante el código pro engineer: Aplicación a un eje ferroviario, 136.

Petráš, R., Škorík, V., \& Polák, J. (2016). Thermomechanical fatigue and damage mechanisms in Sanicro 25 steel. Materials Science and Engineering: A, 650, 52-62. https://doi.org/10.1016/j.msea.2015.10.030

Piovan, M. T. (2014). Capítulo 7. Proyecto y cálculo de ejes y elementos accesorios. In UTN-FRBB. España. 
Shen, Y., Wang, S., Li, X., \& Dhillon, B. S. (2010). Multiaxial fatigue life prediction of kiln roller under axis line deflection. Applied Mathematics and Mechanics, 31(2), 205214. https://doi.org/10.1007/s10483-0100208-X

PL Nuñez, RA Hincapié. (2013), Metodología para Reubicación de Transformadores de Distribución Considerando el Sistema de protección. Revista Colombiana de Tecnologías de Avanzada ISSN: 1692-7257.

Vachon, W. A. (1988). Effects of control algorithms on fatigue life and energy production of vertical axis wind turbines. In American Society of Mechanical Engineers, Solar Energy Division (Publication) SED (Vol. 5, pp. 149-158). ASME. Retrieved from

http://www.scopus.com/inward/record.url?ei $\mathrm{d}=2$-s2.0-0023828031\&partnerID=tZOtx3y1

Waldon, C. A. (1983). Effects of yaw rate on the fatigue life of horizontal axis wecs. (pp. 197202). American Solar Energy Soc Inc. Retrieved from http://www.scopus.com/inward/record.url?ei $\mathrm{d}=2-\mathrm{s} 2.0-0020874905 \&$ partnerID=tZOtx3y1 